Research Paper

\title{
TLR2 Mediates Immunity to Experimental Cysticercosis
}

\author{
José L. Reyes¹, Marisol I. González¹, Yadira Ledesma-Sotoํ, Abhay R. Satoskar² and Luis I. Terrazas ${ }^{1 凶}$
}

1. Unidad de Biomedicina, Facultad de Estudios Superiores-Iztacala, Universidad Nacional Autónoma de México (UNAM), Mexico

2. Departments of Pathology and Microbiology, The Ohio State University, USA.

Corresponding author: Luis I. Terrazas, Unidad de Biomedicina, FES-Iztacala, UNAM. Av. De los Barrios 1, Los Reyes Iztacala, Tlalnepantla, Edo. de Mexico. Mexico 54090. Phone: (5255) 5623-1333 ext 39794. Fax: (5255) 5623-1138. E-mail: literrazas@campus.iztacala.unam.mx

(C) Ivyspring International Publisher. This is an open-access article distributed under the terms of the Creative Commons License (http://creativecommons.org/ licenses/by-nc-nd/3.0/). Reproduction is permitted for personal, noncommercial use, provided that the article is in whole, unmodified, and properly cited.

Received: 2011.09.01; Accepted: 2011.10.01; Published: 2011.10.25

\begin{abstract}
Information concerning TLR-mediated antigen recognition and regulation of immune responses during helminth infections is scarce. TLR2 is a key molecule required for innate immunity and is involved in the recognition of a wide range of viruses, bacteria, fungi and parasites. Here, we evaluated the role of TLR2 in a Taenia crassiceps cysticercosis model. We compared the course of T. crassiceps infection in C57BL/6 TLR2 knockout mice (TLR2 ${ }^{-1 /}$ ) with that in wild type C57BL/6 (TLR2 ${ }^{++}$) mice. In addition, we assessed serum antibody and cytokine profiles, splenic cellular responses and cytokine profiles and the recruitment of alternatively activated macrophages (AAM $\phi s$ ) to the site of the infection. Unlike wild type mice, TLR2 $^{-1-}$ mice failed to produce significant levels of inflammatory cytokines in either the serum or the spleen during the first two weeks of Taenia infection. TLR2 ${ }^{-1-}$ mice developed a Th2-dominant immune response, whereas $\mathrm{TLR2}^{+/+}$mice developed a ThI-dominant immune response after Taenia infection. The insufficient production of inflammatory cytokines at early time points and the lack of ThI-dominant adaptive immunity in TLR2 $2^{-/}$mice were associated with significantly elevated parasite burdens; in contrast, $\mathrm{TLR2}^{+/+}$mice were resistant to infection. Furthermore, increased recruitment of AAM $\phi$ s expressing PD-LI, PD-L2, OX40L and mannose receptor was observed in TLR2 ${ }^{-1-}$ mice. Collectively, these findings indicate that TLR2-dependent signaling pathways are involved in the recognition of $T$. crassiceps and in the subsequent activation of the innate immune system and production of inflammatory cytokines, which appear to be essential to limit infection during experimental cysticercosis.
\end{abstract}

Key words: TLR2, Taenia crassiceps, alternatively activated macrophages, cysticercosis

\section{Introduction}

Pathogen recognition receptors (PRRs) function to rapidly detect pathogen invasion and to control innate immune activation leading to inflammation [1]. The role of innate immunity against bacteria and protozoan parasites has been extensively studied. Specialized PRRs such as Toll-like receptors (TLRs), nucleotide-binding and oligomerization domain (NOD)-like receptors (NLRs), and some C-type lectin receptors (CLRs) recognize specific secreted or membrane-bound components of pathogens called pathogen-associated molecular patterns (PAMPs). Recogni- tion of PAMPs induces the secretion of strong pro-inflammatory cytokines by innate cells such as dendritic cells (DCs), neutrophils and macrophages. These cytokines induce the classical Th1 response observed in such infections, which may render the host resistant [2]. In contrast, the role of PRRs and innate immunity in the response to helminth infections is poorly understood.

TLR2 is involved in the recognition of a wide range of PAMPs derived from bacteria, fungi, parasites and viruses. The PAMPs recognized by TLR2 are 
mainly lipoconjugates such as lipopeptides, lipoproteins, glycolipids, peptidoglycans, lipoteichoic acid, lipoarabinomannan, zymosan and glycosylphosphatidylinositols (GPIs) from Trypanosoma, Leishmania, Toxoplasma, and Plasmodium [3-8]. Activation of DCs and macrophages by TLR2 ligands has been shown to induce both Th1 and Th2 responses, and the polarization of $\mathrm{T}$ cell responses appears to be related to the ligand and to the interaction of TLR2 with other TLRs [9-11]. TLR2 forms heterodimers with TLR1 or TLR6. The TLR2-TLR1 heterodimer recognizes triacylated lipopeptides from Gram-negative bacteria and mycoplasma, whereas the TLR2-TLR6 heterodimer recognizes diacylated lipopeptides from Gram-positive bacteria and mycoplasma [12, 13]. In contrast, although helminths are rich in lipopeptides, glycolipids and phospholipids, the TLR ligands expressed by helminth parasites remain unknown. For example, Schistosoma mansoni, Taenia solium and Echinococcus granulosus [14-19] have been reported to contain such type of molecules, but their role in the immunobiology of such parasites are largely unknown.

Alternative activation of macrophages was first proposed in the early 1990s when Gordon described a novel activation status of macrophages that depended on interleukin (IL)-4, the signature cytokine of the Th2 arm of the immune response [20]. Thereafter, studies of alternatively activated macrophages (AAM $\phi s)$ have focused on helminthic experimental models, as these parasites are strong inducers of Th2 responses. These studies have suggested that AAM $\phi$ s play divergent roles during responses to different helminths. For example, the intestinal nematodes Heligmosomoides bakeri and Nippostrongylus brasiliensis could not be expelled in the absence of AAM $\phi s$, demonstrating an effector role for AAM $\phi s$ in the response to these parasites, reviewed in [21]. In contrast, a recent study has demonstrated that upon infection with Trichuris muris, mice with arginase-deficient macrophages presented a course of infection similar to that observed in wild type mice [22]. Furthermore, during Schistosoma mansoni infection, $\mathrm{AAM} \phi \mathrm{s}$ did not alter parasite numbers; however, increased immunopathology characterized by egg deposition-induced granulomas of the liver was observed in the absence of $\mathrm{AAM} \phi \mathrm{s}$ [23].

We have previously demonstrated that AAM $\phi \mathrm{s}$ with in vitro suppressive capacity infiltrate the peritoneal cavity and facilitate Taenia crassiceps infection [24]. In contrast to observations in other helminth models, we found that an early recruitment of this population was necessary for the progress of this infection [25].
In addition, $T$. crassiceps has proven a useful model to study immunobiological factors associated with resistance and susceptibility to cysticercosis [26-28]. Furthermore, T. crassiceps shares many antigens with the natural parasite causing cysticercosis, $T$. solium $[29,30]$. Some of these shared molecules are of lipid origin [31].

Schistosome-derived products have been shown to bind TLRs on APCs; specifically, TLR2 has been shown to recognize schistosome PAMPs in both human and mice $[32,33]$. Recently, TLR2 and/or TLR3 have been shown to recognize lipid fractions derived from S. mansoni, driving monocyte-derived DCs to induce Th2-skewed responses [34]. This effect is largely mediated through the downregulation of pro-inflammatory/Th1-associated cytokines in lipid-stimulated DCs. Although these observations suggest that TLR2 mediates Th2 responses during schistosomiasis, the role of this receptor in the susceptibility to other helminth infections is unknown.

Here, we investigated the effect of the absence of TLR2 on cytokine production and AAM $\phi$ s recruitment and resistance during T. crassiceps infection. Our findings demonstrate that the loss of TLR2 reduces the production of pro-inflammatory cytokines, resulting in a Th2 bias and significantly impaired resistance to $T$. crassiceps infection.

\section{MATERIALS AND METHODS}

\section{Mice, parasites and infection}

Six- to eight-week-old female TLR2-deficient (TLR2 -/-) and TLR2 wild type (TLR2 +/+) C57BL/6 mice were maintained in FES-Iztacala, UNAM animal facilities according to the Faculty Animal Care and Use Committee and government guidelines (official Mexican regulation NOM-062-ZOO-1999), which are in strict accordance with the recommendations in the Guide for the Care and Use of Laboratory Animals of the National Institutes of Health of the USA. The protocol was approved by the Committee on the Ethics of Animal Experiments of the FES-Iztacala, UNAM. Mice were sacrificed using a $\mathrm{CO}_{2}$ chamber, and all efforts were made to minimize pain.

Metacestodes of $T$. crassiceps were harvested from the peritoneal cavity of female BALB/c mice after 2 to 4 months of infection. The cysticerci were washed four times in sterile phosphate-buffered saline (PBS) (0.15 M, pH 7.2). Because C57BL/ 6 mice are considered resistant to low doses (10 metacestodes) of T. crassiceps, we infected mice with 20 metacestodes to determine whether this resistant strain could be rendered susceptible to this parasite. Experimental infection was achieved by intra-peritoneal (i.p.) injection 
with 20 small (approx. $2 \mathrm{~mm}$ in diameter) non-budding cysticerci of $T$. crassiceps suspended in $0.3 \mathrm{ml}$ of PBS per mouse.

\section{Determination of parasite burden}

To measure parasite burden in the presence or absence of TLR2, mice were sacrificed at 2-week intervals during the 8-week course of infection, and the peritoneal cavities were extensively washed with sterile PBS. Parasites were harvested from the peritoneum and counted in petri dishes. Data are presented as the mean number of parasites per mouse.

\section{Measurement of cytokines in serum (sandwich ELISA)}

At the indicated time points, blood was collected from tails of individual T. crassiceps-infected TLR2-/and TLR2 ${ }^{+/+}$mice. The samples were not pooled. Sera were stored at $-70^{\circ} \mathrm{C}$ until used for cytokine measurement. Kits for the measurement of interleukin (IL)-4, IL-10, IL-12 and interferon (IFN)-gamma were purchased from Peprotech, Mexico. Assays were performed following the manufacturer's instructions.

\section{Quantification of specific anti-Taenia antibod- ies (ELISA)}

In order to determine whether the cytokines worked as expected, circulating specific antibodies anti-Taenia IgG2a and IgG1 isotypes were measured as markers of Th1 and Th2 responses, respectively. Briefly, 96-well Polysorp plates (Nunc, Denmark) were coated with total soluble Taenia antigen $(100 \mu \mathrm{l}$, $10 \mu \mathrm{g} / \mathrm{ml})$. The plates were blocked with $200 \mu \mathrm{l}$ of blocking solution (PBS/BSA 1\%) for $2 \mathrm{~h}$ at $37{ }^{\circ} \mathrm{C}$ and washed. The sera from the mice were serially diluted starting at a dilution factor of 1:50 and added to the plates. Next, the plates were incubated at $37^{\circ} \mathrm{C}$ for $2 \mathrm{~h}$, and serum antibodies were detected using biotinylated and HRP-conjugated rat anti-mouse IgG2a and IgG1 antibodies (Zymed, USA). The substrate (ABTS/ $\mathrm{H}_{2} \mathrm{O}_{2} 10 \%, 100 \mu \mathrm{l} /$ well) was added, and plates were read at $405 \mathrm{~nm}$ in a plate reader (Termo Labsystems). Data are presented as the optical density of the samples diluted 1:50.

\section{Splenocyte cultures}

Proliferation assays were performed on splenocytes obtained from $T$. crassiceps-infected mice 8 weeks after infection. Briefly, single cell suspensions were prepared in RPMI 1640 supplemented with $10 \%$ fetal bovine serum, $100 \mathrm{U} / \mathrm{ml}$ of penicillin-streptomycin, $2 \mathrm{mM}$ glutamine, $25 \mathrm{mM}$ HEPES buffer, and $1 \%$ non- essential amino acids (complete medium) (all from GIBCO BRL, Grand Island, N.Y.).
The erythrocytes were lysed, and viable cells were adjusted to $3 \times 10^{6}$ cells $/ \mathrm{ml}$. The cell suspension (100 $\mu \mathrm{l} /$ well) was placed into 96-well flat-bottomed culture plates (Costar, Cambridge, Mass.), stimulated with TcAg $(50 \mu \mathrm{g} / \mathrm{ml})$ in a total volume of $200 \mu \mathrm{l}$, and incubated at $37^{\circ} \mathrm{C}$ for $96 \mathrm{~h}$. Eighteen hours prior to culture termination, $0.5 \mu \mathrm{Ci}$ of $\left[{ }^{3} \mathrm{H}\right]$-thymidine (185 GBq/mmol) (Amersham, Buckinghamshire, England) was added to each well. The cells were harvested, and thymidine uptake was measured with a Microbeta plate counter (Wallac, Turku, Finland). Values are expressed as mean counts per minute for triplicate wells. Additionally, the supernatants from these cultures were analyzed for IFN- $\gamma$, IL-4, IL-10 and IL-12 production by ELISA (Peprotech, Mexico).

\section{RT-PCR of adherent peritoneal cells}

TLR2 wild type mice and TLR2-deficient mice were sacrificed at different times after infection. Peritoneal exudate cells (PECs) were obtained by peritoneal lavage with $10 \mathrm{ml}$ of ice-cold sterile saline solution (Pisa, Mexico). PECs were washed twice, and red blood cells were lysed by hypotonic shock with ammonium chloride. Following two washes, viable cells (trypan blue exclusion routinely over 95\%) were counted and adjusted to $5 \times 10^{6}$ cells $/ \mathrm{ml}$ in RPMI (Gibco BRL) supplemented with 10\% FCS, 100 U/ml of penicillin/streptomycin, $2 \mathrm{mM}$ glutamine, $25 \mathrm{mM}$ HEPES buffer, and $1 \%$ non-essential amino acids (all from GIBCO, BRL Grand Island, NY) and cultured in six-well plates (Costar, Cambridge, MA, USA). After 2 $\mathrm{h}$ of culture at $37^{\circ} \mathrm{C}$ and $5 \% \mathrm{CO}_{2}$, non-adherent cells were removed by vigorous washing with warm supplemented RPMI medium. Adherent cells were lysed with TRIzol reagent (Invitrogen, USA), and total RNA was extracted using the chloroform-isopropanol technique. Next, $5 \mu \mathrm{g}$ of RNA was reverse transcribed to prepare cDNA using the first strand synthesis superscript II kit (Invitrogen, USA). cDNA samples were standardized based on the content of GAPDH cDNA. PCR was performed in a total volume of $50 \mu \mathrm{l}$ in PCR buffer in the presence of $0.2 \mathrm{M} \mathrm{dNTP}, 0.2 \mathrm{mM}$ of each primer, and $2.5 \mathrm{U}$ of Platinum Taq DNA polymerase (Invitrogen, CA, USA) using a Palmcycler (Corbett Research, Australia). After 35 cycles of amplification, the PCR products were separated by electrophoresis on a $1.5 \%$ agarose gel and visualized by Sybr Green staining. Sequences and conditions for PCR of Resistin like molecule alpha (RELM $\alpha)$, chitinase Ym1 and arginase-1 were previously reported [35].

\section{Flow cytometry of total peritoneal exudate cells (PECs)}

The expression of membrane markers on total 
peritoneal cells was analyzed by flow cytometry. Total PECs were blocked with an anti-mouse FcyR antibody (CD16/CD32) for $15 \mathrm{~min}$ and stained for an additional $30 \mathrm{~min}$ with an allophycocyanin (APC)-conjugated monoclonal antibody specific for mouse F4/80, phycoerythrin (PE)-conjugated antibodies specific for PDL1, PDL2, or OX40L, or a fluorescein isothiocyanate (FITC)-conjugated antibody specific for MMR in FACS buffer (PBS containing $0.5 \%$ FBS and $0.1 \% \mathrm{NaN}_{3}$ ). All antibodies were purchased from Biolegend (San Diego, CA USA). Stained cells were analyzed using a FACSCalibur flow cytometer and Cell Quest software (Becton Dickinson). Live cells were electronically gated using forward and side scatter parameters.

\section{Statistical analysis}

The TLR2 ${ }^{+/+}$and TLR2\%-- groups considered in this study were compared using the Student's unpaired t-test. A $P$ value $<0.05$ was considered significant.

\section{Results}

The absence of TLR2 favors Taenia crassiceps infection. TLR2 is one of the most studied receptors in the context of infections with pathogens ranging from virus to fungi $[36,37]$, but the mechanisms underlying TLR-mediated antigen recognition and regulation of immune responses and the role of TLR2 in helminth parasitic diseases are not completely understood. The purpose of this study was to determine the role of TLR2-mediated signaling in the outcome of murine cysticercosis caused by the extra-intestinal helminth T. crassiceps. To address this question, we compared the course of $T$. crassiceps infection in TLR2-/-C57BL/6 mice (TLR2/-) with that in wild type C57BL/6 $\left(\mathrm{TLR}^{+/+}\right)$mice. In addition, we assessed serum antibody and cytokine profiles, splenic cellular responses and cytokine profiles and the recruitment of AAM $\phi \mathrm{s}$ to the site of infection.

Age-matched TLR2 ${ }^{+/+}$and TLR2 $/-$female mice were i.p. infected with 20 cysticerci, and the infections were followed for 8 weeks. Both strains of mice displayed a slow but progressive increase in parasite numbers in their peritoneal cavities, with comparable parasite burdens 2 and 4 weeks post-infection (Fig. 1). Interestingly, as the infection progressed, parasite burdens were significantly higher in TLR2 $\%$ mice than in TLR2 ${ }^{+/+}$mice, which successfully controlled the infection by week 6 post-infection (Fig. $1 ; \mathrm{p}<0.01$ ). Thus, at week 8 post-infection, TLR2 $/$ - mice were significantly more susceptible to experimental cysticercosis.

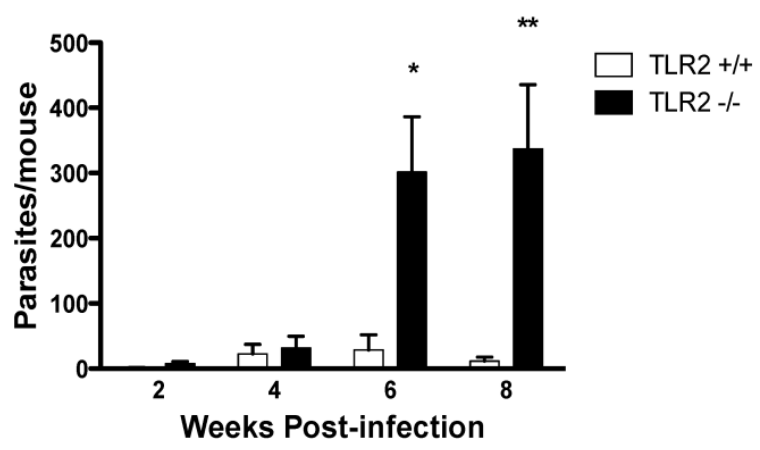

Figure I. TLR2 ${ }^{-/-}$C57BL/6 mice are unable to efficiently control Taenia crassiceps infection. The course of i.p. T. crassiceps infection in TLR2 ${ }^{-/-}$and $T L R 2^{+/+}$mice following infection with 20 cysticerci. Data are expressed as means \pm SE of data from 4-6 mice per group. $* P<0.05$ and $* * P<0.0$ I when comparing TLR2 ${ }^{-/-}$mice to TLR2 ${ }^{+/+}$mice at the same time point of infection. Similar results were observed in two independent experiments.

TLR2 deficiency modifies the antibody response to T. crassiceps. Next, we measured the levels of Th1-associated IgG2a and Th2-associated IgG1 antibodies in TLR2 $/-$ and TLR2 $^{+/+}$mice at different time points following infection with $T$. crassiceps. Whereas both strains displayed similar production of anti-Taenia-specific IgG2a during the course of infection (Fig. 2A), the anti-Taenia specific IgG1 response was quite different. At weeks 2 and 4 post-infection, $T$. crassiceps-infected TLR2 ${ }^{+/+}$and TLR2 $/$-mice displayed comparable titers of anti-Taenia-specific Th2-associated IgG1, but as infection progressed, anti-Taenia-specific IgG1 levels were significantly higher in TLR2 $\%$ mice. The most significant difference between TLR2 $\%$ and TLR2 ${ }^{++}$mice was observed at week 8 p.i. (Fig. 2B). These observations support the findings of previous studies that demonstrated that $T$. crassiceps-susceptible mouse strains showed increased production of IgG1 $[38,39]$.

TLR2\% mice display overproduction of Th2 cytokines in response to $T$. crassiceps infection. To investigate the potential involvement of TLR2 in the acquired immune response following $T$. crassiceps infection, splenocytes were stimulated with $T$. crassiceps soluble antigen (TcAg) and Con A. The spleen cells from T. crassiceps-infected TLR2 ${ }^{+/+}$and TLR2 $-{ }^{-}$ mice displayed different patterns of proliferative responses following in vitro stimulation with $T$. crassiceps antigen. During the early phase of infection, TcAg-stimulated splenocytes from TLR2 ${ }^{+/+}$mice displayed significantly higher proliferative responses than those from TLR2-/- mice, and TLR2 ${ }^{+/+}$splenocytes displayed significantly stronger responses during chronic infection (Fig. 3A, p<0.05). 


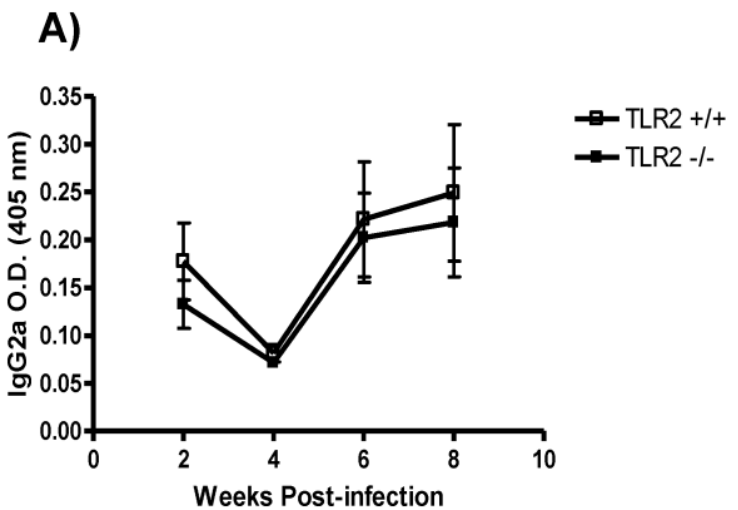

B)

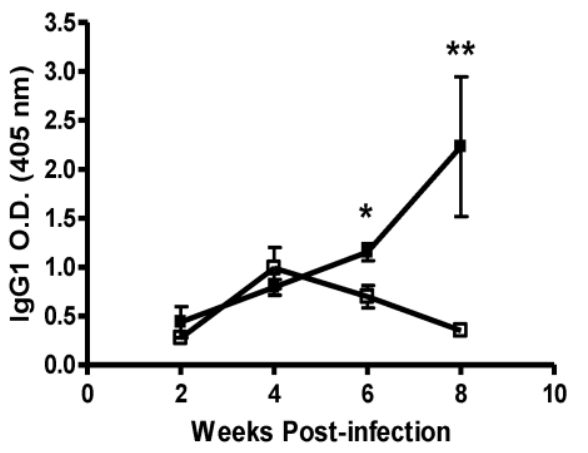

Figure 2. Kinetics of antibody production during T. crassiceps infection in TLR2 ${ }^{-/}$(closed squares) and TLR2 ${ }^{+/+}$(open squares) mice. A) Anti-T. crassiceps lgG2a. B) Anti-T. crassiceps lgGI. Sera were obtained from the tail vein of each mouse at the indicated time points. ELISA plates were coated with $0.5 \mu \mathrm{g} /$ well of $T$. crassiceps cysticerci soluble extract. Data are presented as means $\pm S E\left(n=4\right.$ animals) and are representative of two independent experiments. ${ }^{*} P<0.05$ when comparing $\mathrm{TLR2}^{-/-}$mice to $\mathrm{TLR2}^{+/+}$mice at the same time point.

A)

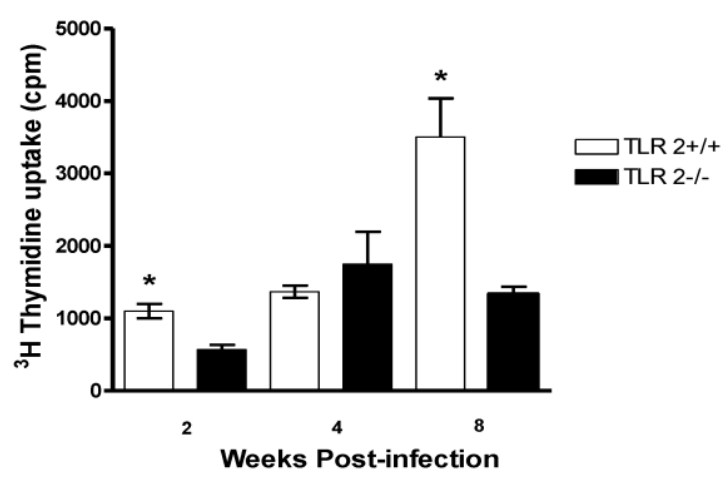

C)

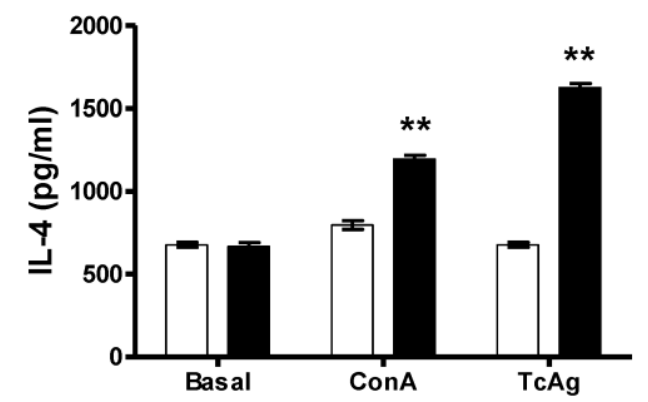

B)

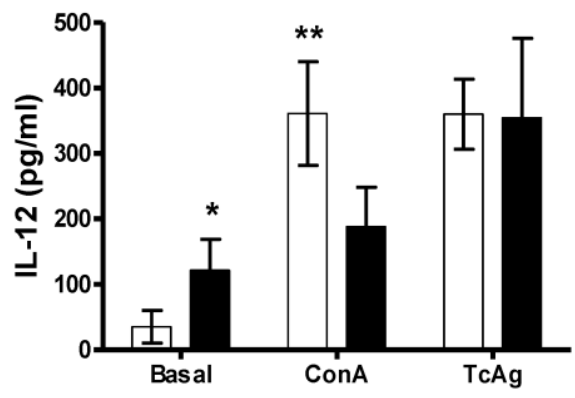

D)

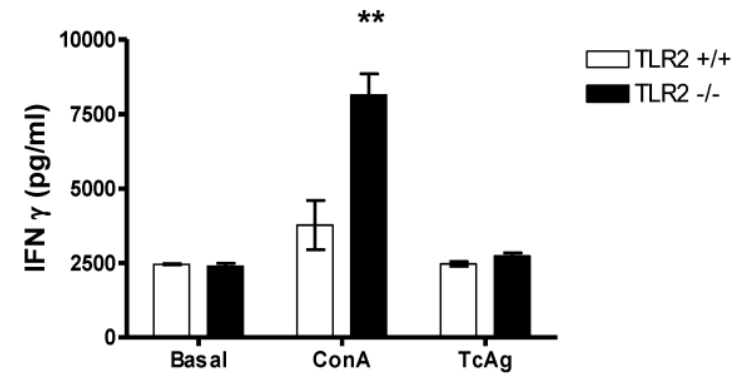

Figure 3. Cell proliferation and Th1-/Th2-related cytokine production in infected TLR2 ${ }^{-/ 2}$ and wild-type mice. A) Proliferative responses were assessed by measuring ${ }^{3} \mathrm{H}$-thymidine incorporation in splenocytes stimulated with soluble Taenia crassiceps antigen ( $\mathrm{TcAg}$ ) and Concanavalin $\mathrm{A}(\mathrm{ConA})$ at different time points post-infection. Splenocytes from mice infected with Taenia crassiceps for 8 weeks were stimulated with TcAg or ConA in vitro. Production of (B) IL-I2, (C) IL-4 and (D) IFN $-\gamma$ was quantified by ELISA. Data are expressed as means \pm SEM and are representative of two independent experiments with similar results. $* P<0.05$ and $* * P<0.01 . \mathrm{n}=4-6$ mice per group. TLR, Toll-like receptor. 
Next, supernatants from the same cell cultures were evaluated for both antigen-specific and Con-A-induced cytokine production. At week 8 post-infection, TcAg-stimulated splenocytes from TLR2 ${ }^{+/+}$mice produced significantly higher levels of Th1-associated IL-12 in response to Con-A than those from TLR2 $\%$ mice (Fig. 3B). In contrast, TcAg-stimulated splenocytes from TLR2 $\%$ mice produced significantly more IL-4 than those from $\mathrm{TLR}^{+/+}$mice; similar results were observed when the cells were stimulated with Con-A (Fig. 3C). These findings demonstrate the ability of TLR2 $\%$ mice to maintain a sustained Th2-type response. In contrast, both strains displayed comparable antigen-specific IFN- $\gamma$ responses (Fig. 3D).

Enhanced systemic Th2 cytokine production in TLR2 $\%$ mice. To address the impact of the absence of TLR2 on the regulation of cytokine production, we analyzed circulating cytokine levels as a measure of the general status of the immune response. IFN- $\gamma$ lev-

A)

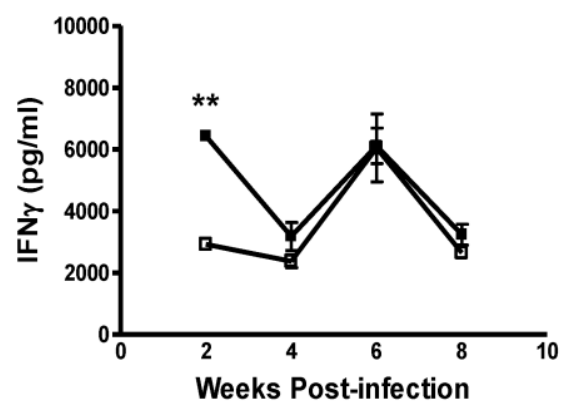

C)

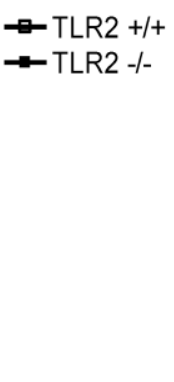

)

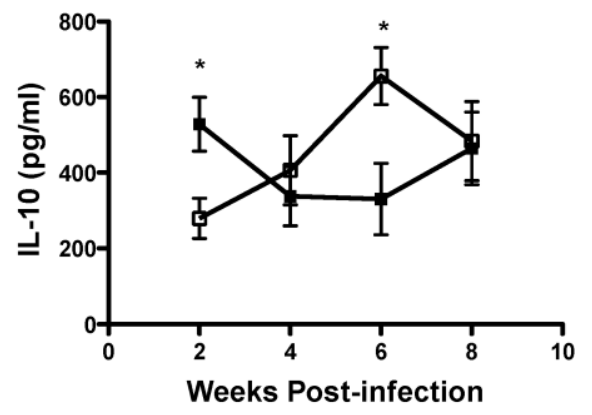

els in sera were initially higher in TLR2/- mice, but as the infection progressed, similar levels of IFN- $\gamma$ were detected in both mouse strains (Fig. 4A). In sharp contrast, T. crassiceps-infected TLR2 $\%$ - mice produced higher levels of IL- 4 than their TLR2 ${ }^{+/+}$counterparts even at the earliest point time assayed (Fig. 4B). This finding suggests a possible Th2 cytokine imbalance, which has been shown to be permissive for T. crassiceps growth [26]. In addition, IL-10 production peaked at an early time point in the TLR2 $\%$ group, and by 6 weeks p.i., less IL-10 was detected in TLR2-\% mice than in TLR2 ${ }^{+/+}$mice; however, by the end of the infection, IL-10 levels were similar in both mouse strains (Fig. 4C). As previously observed in cell culture, IL-12 production was highly dependent on TLR2 signaling, as the absence of TLR2 resulted in decreased levels of IL-12 at 2 and 6 weeks after infection (Fig. 4D). Together, these data demonstrate that an increased parasite burden is associated with elevated levels of Th2 cytokines.

B)

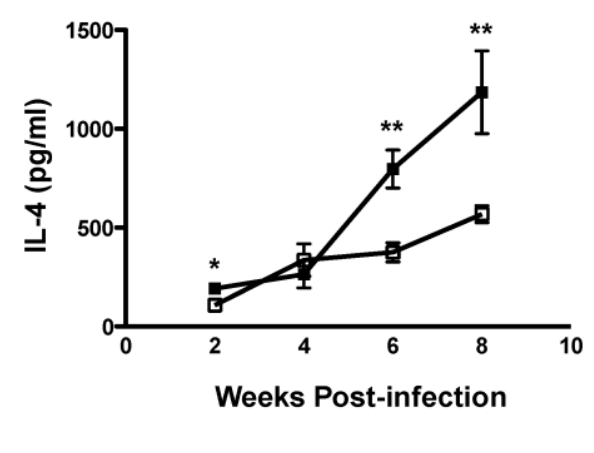

D)

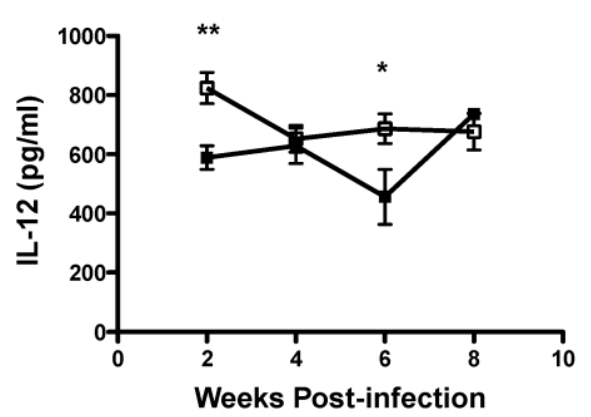

Figure 4. Systemic Th2-biased responses in TLR2 ${ }^{-1-}$ mice infected with Taenia crassiceps metacestodes. The kinetics of cytokine production after infection with 20 metacestodes was assessed by measuring (A) IFN- $\gamma$ detection in sera. (B) TLR2 $2^{-1-}$ mice displayed increased IL-4 levels as the infection progressed; (C) IL-I0 and (D) IL-I 2 levels in sera were inconsistent. The data shown are representative of two independent experiments, with four mice per group. The error bars indicate standard deviations. $* P<0.05$ and $* * P<0.01$. $n=4-6$ mice per group. TLR, Toll-like receptor. 
Increased recruitment of $A A M \phi s$ to the peritoneal cavity following $T$. crassiceps infection in TLR2\% mice. We previously reported that T. crassiceps infection results in the recruitment of a large population of (AAM $\phi s)$ with suppressive capacity [40, 41]. Here, we examined the effect of TLR2 deficiency on macrophage polarization. First, we performed RT-PCR to detect the expression of genes associated with suppressive AAM $\phi$ s, such as Relm- $\alpha$, Ym-1, Arginase-1, PDL1 and PDL2, at different time points during the 8-week infection. We found that in addition to the low levels of parasite proliferation observed in $\mathrm{TLR}^{+/+}$mice, the expression of these markers did not increase in $\mathrm{TLR}^{+} /+$adherent macrophages (Fig. 5). In contrast, as the time of infection advanced, the expression of AAM $\phi$ markers progressively increased in macrophages obtained from TLR2-/- animals, and Relm- $\alpha$, Arginase- 1 and PDL1 were the first markers that appeared at week 6 of infection. Analysis at 8 weeks of infection showed that macrophages from TLR2 $/$ - mice expressed all of the markers associated with alternative activation (Relm- $\alpha^{+}, \mathrm{Ym}-1^{+}$, Arginase- $1^{+}, \mathrm{PDL}^{+}, \mathrm{PDL}^{+}$, iNOS-), suggesting an increase in the recruitment of AAM $\phi s$ in the absence of TLR2 and an important role for TLR2 in macrophage polarization (Fig. 5).

In addition to RT-PCR, peritoneal macrophages were assessed by flow cytometry to confirm the sur- face expression of the molecules associated with alternative activation. Figure $6 \mathrm{~A}$ shows representative dot plots and the analysis of $\mathrm{F} 4 / 80^{+} \mathrm{MR}^{+}$cells in the peritoneal cavities of both groups of mice. Comparable percentages of these cells were observed $2 \mathrm{wk}$ post-infection in both $\mathrm{TLR}^{+/+}$and $\mathrm{TLR} 2^{-/-}$mice; however, during the chronic phase of infection (6 to 8 weeks), up to $58 \%$ of the $\mathrm{F} 4 / 80^{+}$cells recovered from TLR2 $/-$ mice expressed MR, whereas only $21 \%$ of F4 $/ 80^{+}$cells from TLR2 ${ }^{+/}$mice expressed MR. Moreover, flow cytometry analysis of PDL1 and PDL2 expression on $\mathrm{F} 4 / 80^{+}$cells demonstrated similar patterns of expression, with more than double the number of TLR2/- macrophages expressing each marker 6 and 8 weeks after infection (Fig. 6B-C). Together, these results confirm that the hallmarks of alternative activation were more prevalent in TLR2/- macrophages than in macrophages with intact TLR2 signaling. Finally, we analyzed the expression of OX40L, which is a co-stimulatory molecule involved in Th2 polarization; we found that TLR2-/- macrophages also over expressed OX40L on their surface (Fig. 6D), and $50 \%$ fewer $\mathrm{F} 4 / 80^{+} \mathrm{OX} 40 \mathrm{~L}^{+}$macrophages were observed in TLR2 ${ }^{+/+}$mice. Hence, using multiple approaches, we have demonstrated that TLR2 deficiency causes sustained alternative activation in macrophages, potentially supporting $T$. crassiceps establishment.

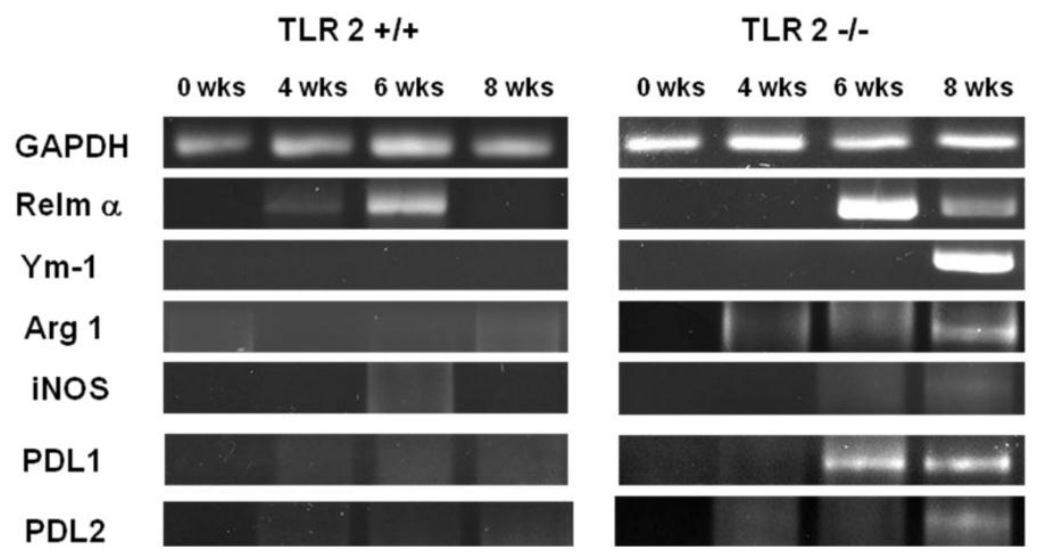

Figure 5. Taenia crassiceps infection induces peritoneal recruitment of AAMФs after 8 weeks of infection in TLR2 ${ }^{-/-}$mice. RT-PCR analysis of adherent peritoneal macrophages recovered from animals infected with 20 metacestodes $0,4,6$ and 8 weeks p.i. Increased expression of genes associated with AAMФs, such as Arginase I, RELM- $\alpha$, Ym I, PD-LI and PD-L2, was detected only in TLR2 ${ }^{--}$T. crassiceps-infected mice. The data shown are representative of two independent experiments with four mice per group. 
A)
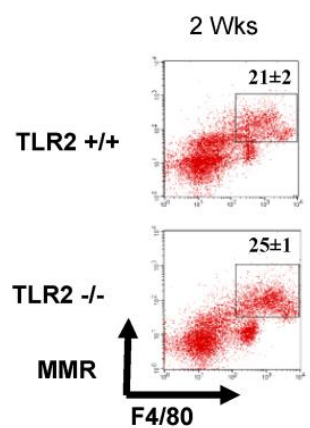

B)
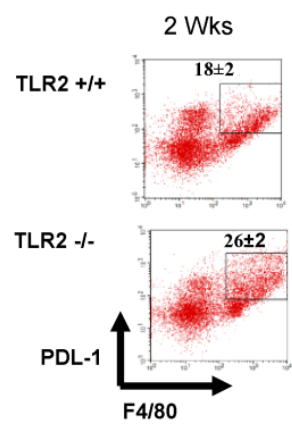

C)
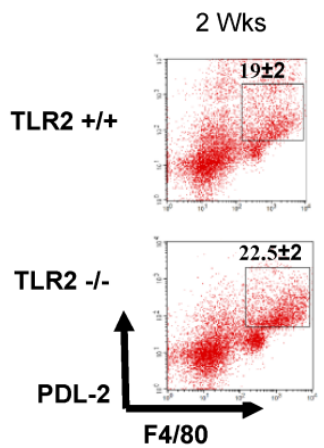

D)

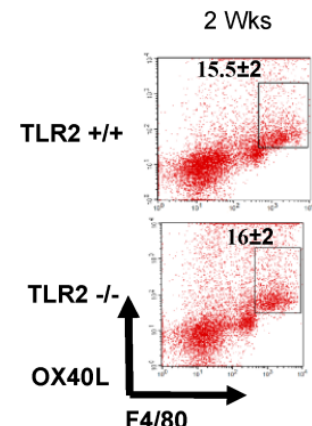

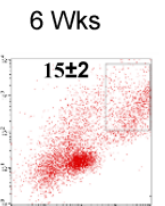
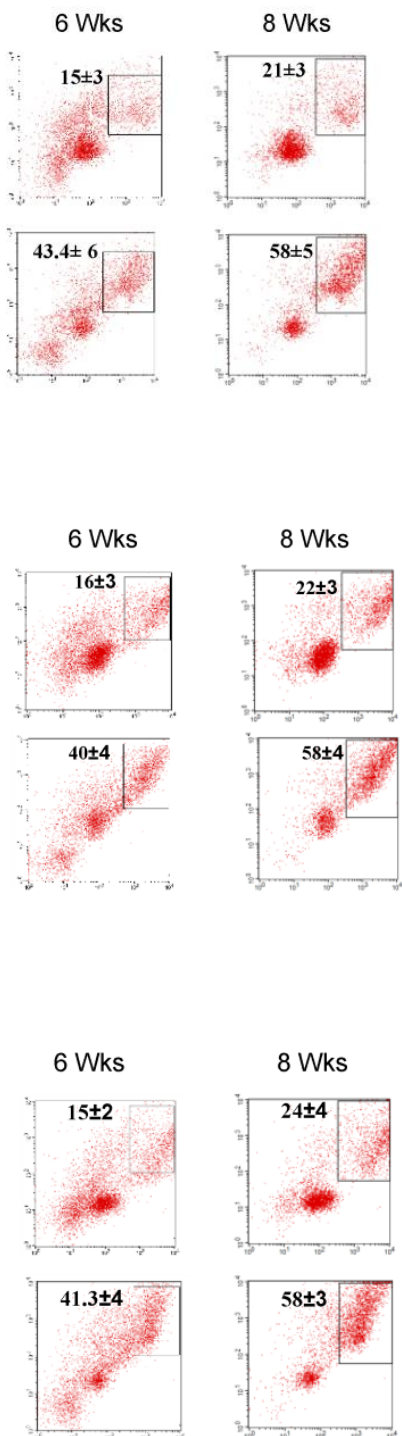

8 Wks
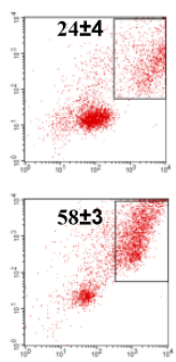

8 Wks
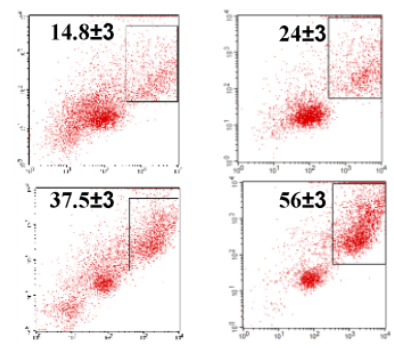
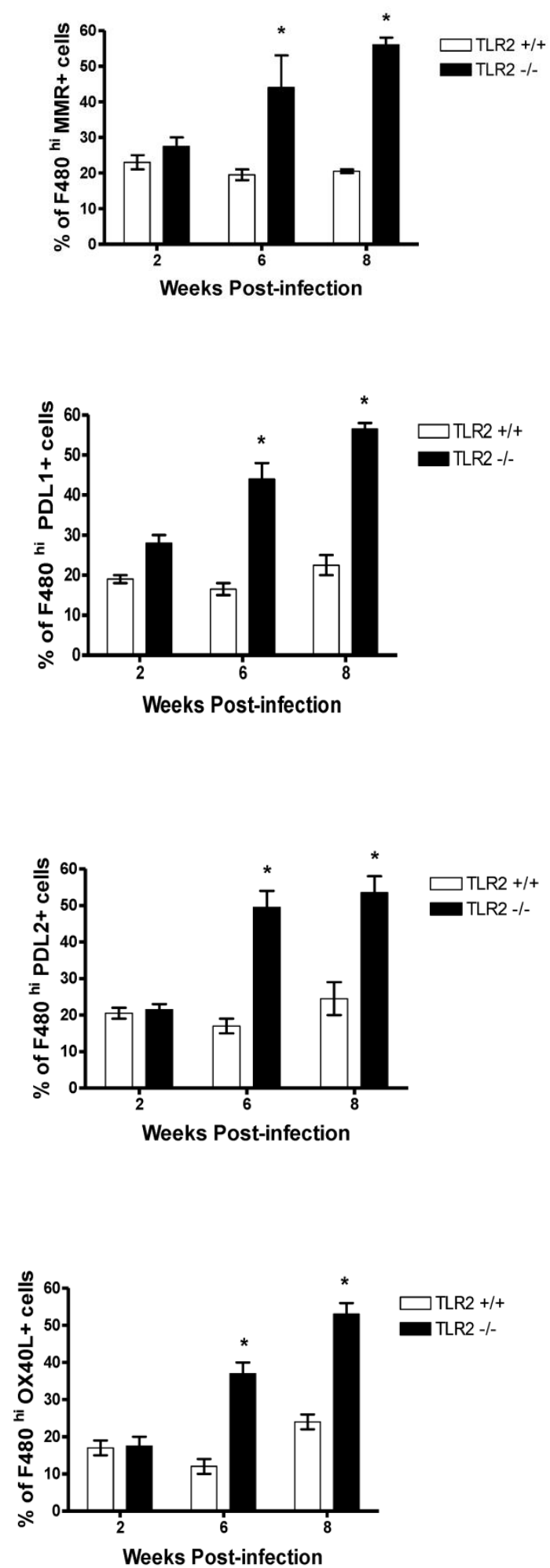

Figure 6. Taenia crassiceps infection induces peritoneal recruitment of AAMФs after 8 weeks of infection only in TLR2 $2^{-1-}$ mice. Flow cytometric analysis of peritoneal exudate cells recovered from animals infected with 20 metacestodes 8 weeks p.i. (A) Surface expression of F4/80 and MMR (CD206). (B) Surface expression of F4/80 and PDL-I, (C) F4/80 and PDL-2 and (D) F4/80 and OX40L. The dot plots represent four mice per group, and the data are representative of two independent experiments. 


\section{Discussion}

Innate immune receptors, including TLR2 and CLRs, play important roles in the establishment of innate responses to infection and provide signals to initiate and shape the adaptive immune response [1]. Similar to other helminth infections, the innate mechanisms that initiate or contribute to the immune response against cysticercosis are largely unknown; however, toll-like receptors (TLRs) appear to be important in recognizing these parasites $[32,42]$. The involvement of TLRs in experimental or natural cysticercosis has begun to be elucidated in laboratory models. For example, an increase in the expression of several TLRs, such as TLR4 and TLR2, has been observed in murine NCC caused by Mesocestoides corti. This increase in TLR expression may be related to resistance, given that MyD88-deficient mice are susceptible to NCC [43, 44]. Fewer studies have addressed these questions in humans, but a recent study of NCC patients and healthy controls demonstrated an association between a TLR4 polymorphism and an increased risk of NCC, as subjects with a TLR4 (Asp299Gly or Thr399Ile) polymorphism were more susceptible to NCC than control subjects [45]. Moreover, among patients with NCC, the presence of both Asp299Gly and Thr399Ile polymorphisms was associated with increased severity of NCC. In the present study, we demonstrated that TLR2 contributes to resistance to $T$. crassiceps infection by regulating type 2 immune responses, including the production of antigen-specific IgG1 and IL-4, and by controlling AAM $\phi$ recruitment. All of these immunological elements have been associated with susceptibility to T. crassiceps cysticercosis [35, 38, 46]. Moreover, our findings suggest, for the first time, that TLR2-mediated signaling is critically required to control parasite growth in cysticercosis, in particular, and in helminth infection, in general. Variations in TLRs may thus result in altered or defective TLR-mediated signaling that, in turn, may adequately direct the immune response against this parasite.

In addition to $\mathrm{AAM} \phi \mathrm{s}$, other regulatory subpopulations have also been described in helminth infections, such as Foxp3 ${ }^{+}$regulatory T cells. In our T. crassiceps experimental model, we did not detect this $\mathrm{T}$ cell population in the spleen or mesenteric lymph nodes of heavily parasitized C57BL/ 6 mice at least at 8 weeks after infection; however, we cannot rule out the presence of Foxp3 ${ }^{+} \mathrm{T}$ cells in T. crassiceps infection at earlier times of infection or in the peritoneal cavity where this cestode resides. Further studies are thus necessary to determine whether the absence of TLR2 affects the regulatory $\mathrm{T}$ cell population.

Previous studies have demonstrated that TLR2-mediated signaling favors the development of protective immunity against intracellular parasites such as L. mexicana, T. gondii and T. cruzi by favoring Th1 development and inflammatory responses [5-7]. During infections with these organisms, TLR2 recognized GPIs, resulting in a pro-inflammatory response associated with the clearance of these protozoa. Helminths also express lipid conjugates either on their surface or as secreted or excreted products. For example, adult worms and eggs from $S$. mansoni express lipids including lysophosphatidylcholine, which has been reported to be sensed by TLR2. This interaction is associated with eosinophil recruitment and activation; however, in clear contrast with our data, the absence of TLR2 reduced the production of Th2-related cytokines and increased the survival rates of experimental schistosomiasis [17]. Interestingly, TLR2 seems to play a similar role during $S$. japonicum infection, as the number of eggs in the liver, which is directly associated with the pathology of this infection, was reduced in TLR2 $\%$ mice [47].

Furthermore, previous studies have showed that T. crassiceps carbohydrates may interact with TLR4, resulting in production of inflammatory cytokines such as IL-6 [48]. Our data suggest that the recognition of lipid molecules through TLR2 might trigger different signaling events, as circulating IL-6 levels were increased in TLR2 $\%$ mice only during the chronic phase of infection ( $8 \mathrm{wks}$ ) and as the levels of IL-6 did not decrease as expected. In contrast, molecules derived from T. solium did not require TLR2 or TLR4 to induce chemokine production in human lymphocytes [49].

Expression of different kind of lipids, such as neutral lipids $(63.9 \%)$, glycolipids $(6.2 \%)$ and phospholipids $(29.9 \%)$, has also been reported in the cestodes T. solium and T. crassiceps [31], but the role of these lipids as PAMPs has not been yet evaluated. Interestingly, both $T$. solium and $T$. crassiceps metacestodes expressed the glycolipid GSL-1; this glycolipid is similar in both species and appears to be the major lipid expressed in these parasites. Importantly, $T$. crassiceps metacestodes also express lysophosphatidylcholine [50], the same molecule shown to be recognized by TLR2 in schistosomiasis [17]. These data suggest that the host may use a similar mechanism to sense two different helminths, but the outcome of recognition of these parasites may be different. In schistosomiasis, the absence of TLR2 appears to ameliorate the pathology and increase host survival $[17,34]$, whereas in experimental cysticerco- 
sis, TLR2 seems to play a very different role, as TLR2-/- mice were highly susceptible to this infection and were unable to stop parasite growth. The disparate outcomes of these two helminth infections in the absence of TLR2 may be related to the ability of TLR2 to form heterodimers with both TLR1 and TLR6 [2]. Parasite recognition by TLR2/1 heterodimers or by TLR2/ 6 heterodimers likely results in the activation of different signaling pathways, raising the interesting question of which heterodimer functions in each infection. Taken together, these studies suggest that hosts may use similar mechanisms to sense helminths and that lysophosphatidylcholine may be one of the first helminth-associated PAMPs for which a putative receptor has been identified (TLR2). Further assays with purified molecules from both $T$. solium and $T$. crassiceps are necessary to confirm this hypothesis in cysticercosis.

In conclusion, our findings demonstrate, for the first time, that TLR2-dependent pathways are involved and play an important immunomodulatory role in resistance to experimental cysticercosis caused by $T$. crassiceps.

\section{Acknowledgments}

This work was supported by grant IN212909 from PAPIIT-UNAM and Grant 23 from PAPCA-Iztacala 2010-2011. MIG and JLR were supported by a fellowship from CONACYT-Mexico. We thank MVZ Leticia Flores and Tomas Villamar for providing excellent care to the animals.

\section{Conflict of Interests}

The authors have declared that no conflict of interest exists.

\section{References}

1. Medzhitov R. Recognition of microorganisms and activation of the immune response. Nature, 2007. 449(7164): 819-26.

2. Kawai $\mathrm{T}$ and Akira S. The roles of TLRs, RLRs and NLRs in pathogen recognition. Int Immunol, 2009. 21(4): 317-37.

3. Bhattacharya $\mathrm{P}$, et al. Arabinosylated lipoarabinomannan-mediated protection in visceral leishmaniasis through up-regulation of toll-like receptor 2 signaling: an immunoprophylactic approach. J Infect Dis, 2010. 202(1): 145-55.

4. Campos M.A, et al. Impaired production of proinflammatory cytokines and host resistance to acute infection with Trypanosoma cruzi in mice lacking functional myeloid differentiation factor 88. Immunol J, 2004. 172(3): 1711-8.

5. Ropert C and Gazzinelli R.T. Regulatory role of Toll-like receptor 2 during infection with Trypanosoma cruzi. J Endotoxin Res, 2004. 10(6): 425-30.

6. Becker I, et al. Leishmania lipophosphoglycan (LPG) activates NK cells through toll-like receptor-2. Mol Biochem Parasitol, 2003. 130(2): 65-74.

7. Debierre-Grockiego F, et al. Activation of TLR2 and TLR4 by glycosylphosphatidylinositols derived from Toxoplasma gondii. Immunol J, 2007. 179(2): 1129-37.
8. Krishnegowda G, et al. Induction of proinflammatory responses in macrophages by the glycosylphosphatidylinositols of Plasmodium falciparum: cell signaling receptors, glycosylphosphatidylinositol (GPI) structural requirement, and regulation of GPI activity. J Biol Chem, 2005. 280(9): 8606-16.

9. Bansal K, et al. Src homology 3-interacting domain of Rv1917c of Mycobacterium tuberculosis induces selective maturation of human dendritic cells by regulating PI3K-MAPK-NF-kappaB signaling and drives Th2 immune responses. J Biol Chem, 2010. 285(47): 36511-22.

10. Jung T.Y, et al. Ursolic acid isolated from Uncaria rhynchophylla activates human dendritic cells via TLR2 and/or TLR4 and induces the production of IFN-gamma by CD4+ naive $\mathrm{T}$ cells. Eur Pharmacol J, 2010. 643(2-3): 297-303.

11. Makino $\mathrm{M}$, et al. Innate immunity in DNA vaccine with Toxoplasma gondii-heat shock protein 70 gene that induces activation DC and Th1 polarization. Vaccine, 2011. 29(10): 1899-905.

12. Shimizu T, Kida Y, and Kuwano K. Triacylated lipoproteins derived from Mycoplasma pneumoniae activate nuclear factor-kappaB through toll-like receptors 1 and 2. Immunology, 2007. 121(4): 473-83.

13. Azuma $M$, et al. The peptide sequence of diacyl lipopeptides determines dendritic cell TLR2-mediated NK activation. PLoS One, 2010. 5(9): e12550.

14. van der Kleij D, et al. A novel host-parasite lipid cross-talk. Schistosomal lyso-phosphatidylserine activates toll-like receptor 2 and affects immune polarization. J Biol Chem, 2002. 277(50): 48122-9.

15. Koster B and Strand M. Schistosoma mansoni: $\operatorname{Sm} 23$ is a transmembrane protein that also contains a glycosylphosphatidylinositol anchor. Arch Biochem Biophys, 1994. 310(1): 108-17.

16. van Die I, et al. Structural and functional analysis of glycosphingolipids of Schistosoma mansoni. Methods Enzymol, 2010. 480: 117-40.

17. Magalhaes K.G, et al. Schistosomal-derived lysophosphatidylcholine are involved in eosinophil activation and recruitment through Toll-like receptor-2-dependent mechanisms. J Infect Dis, 2010. 202(9): 1369-79.

18. Lopez-Marin L.M, et al. Structure and antigenicity of the major glycolipid from Taenia solium cysticerci. Mol Biochem Parasitol, 2002. 119(1): 33-42.

19. Mamuti W, et al. Recent advances in characterization of Echinococcus antigen B. Parasitol Int, 2006. 55: S57-62.

20. Gordon $S$ and Martinez F.O. Alternative activation of macrophages: mechanism and functions. Immunity, 2010. 32(5): 593-604.

21. Reyes J.L and Terrazas L.I. The divergent roles of alternatively activated macrophages in helminthic infections. Parasite immunology, 2007. 29(12): 609-19.

22. Bowcutt $R$, et al. Arginase-1-expressing macrophages are dispensable for resistance to infection with the gastrointestinal helminth Trichuris muris. Parasite immunology, 2011. 33(7): 411-20.

23. Herbert D.R, et al. IL-10 and TGF-beta redundantly protect against severe liver injury and mortality during acute schistosomiasis. Journal of immunology, 2008. 181(10): 7214-20.

24. Terrazas L.I, et al. Role of the programmed Death-1 pathway in the suppressive activity of alternatively activated macrophages in experimental cysticercosis. International journal for parasitology, 2005. 35(13): 1349-58.

25. Reyes J.L, et al. Early removal of alternatively activated macrophages leads to Taenia crassiceps cysticercosis clearance in vivo. International journal for parasitology, 2010. 40(6): 731-42.

26. Terrazas L.I, et al. Shift from an early protective Th1-type immune response to a late permissive Th2-type response in mu- 
rine cysticercosis (Taenia crassiceps). Parasitol J, 1998. 84(1): 74-81.

27. Terrazas L.I, et al. Th1-type cytokines improve resistance to murine cysticercosis caused by Taenia crassiceps. Parasitol Res, 1999. 85(2): 135-41.

28. Rodriguez-Sosa M, et al. Cutting edge: susceptibility to the larval stage of the helminth parasite Taenia crassiceps is mediated by Th2 response induced via STAT6 signaling. Immunol J, 2002. 168(7): 3135-9.

29. Ishida M.M, et al. Helminth antigens (Taenia solium, Taenia crassiceps, Toxocara canis, Schistosoma mansoni and Echinococcus granulosus) and cross-reactivities in human infections and immunized animals. Acta Trop, 2003. 89(1): 73-84.

30. Arruda G.C, et al. Evaluation of Taenia solium and Taenia crassiceps cysticercal antigens for the serodiagnosis of neurocysticercosis. Trop Med Int Health, 2005. 10(10): 1005-12.

31. Baumeister $S$, et al. Comparative serological reactivity of Taenia crassiceps, Taenia solium and Taenia saginata metacestode neutral glycolipids to infection serum from Taenia crassiceps-infected mice. Mol Biochem Parasitol, 1992. 53(1-2): 53-61.

32. Goh F, et al. Selective induction of the Notch ligand Jagged-1 in macrophages by soluble egg antigen from Schistosoma mansoni involves ERK signalling. Immunology, 2009. 127(3): 326-37.

33. van Riet E, et al. Combined TLR2 and TLR4 ligation in the context of bacterial or helminth extracts in human monocyte derived dendritic cells: molecular correlates for Th1/Th2 polarization. Immunol BMC, 2009. 10: 9.

34. Vanhoutte F, et al. Toll-like receptor (TLR)2 and TLR3 sensing is required for dendritic cell activation, but dispensable to control Schistosoma mansoni infection and pathology. Microbes Infect, 2007. 9(14-15): 1606-13.

35. Reyes J.L, et al. Differential response of antigen presenting cells from susceptible and resistant strains of mice to Taenia crassiceps infection. Infect Genet Evol, 2009. 9(6): 1115-27.

36. Yokota $S$, Okabayashi $\mathrm{T}$, and Fujii N. The battle between virus and host: modulation of Toll-like receptor signaling pathways by virus infection. Mediators Inflamm, 2010. 2010: 184328.

37. Roeder A, et al. Toll-like receptors and innate antifungal responses. Trends Microbiol, 2004. 12(1): 44-9.

38. Rodriguez-Sosa $M$, et al. Macrophage migration inhibitory factor plays a critical role in mediating protection against the helminth parasite Taenia crassiceps. Infect Immun, 2003. 71(3): 1247-54.

39. Rodriguez-Sosa $\mathrm{M}$, et al. Altered $\mathrm{T}$ helper responses in CD40 and interleukin-12 deficient mice reveal a critical role for Th1 responses in eliminating the helminth parasite Taenia crassiceps. Int Parasitol J, 2003. 33(7): 703-11.

40. Rodriguez-Sosa $\mathrm{M}$, et al. Chronic helminth infection induces alternatively activated macrophages expressing high levels of CCR5 with low interleukin-12 production and Th2-biasing ability. Infect Immun, 2002. 70(7): 3656-64.

41. Terrazas L.I, et al. Role of the programmed Death-1 pathway in the suppressive activity of alternatively activated macrophages in experimental cysticercosis. Int Parasitol J, 2005. 35(13): 1349-58.

42. He $\mathrm{Y}$, et al. Recombinant Ov-ASP-1, a Th1-biased protein adjuvant derived from the helminth Onchocerca volvulus, can directly bind and activate antigen-presenting cells. Immunol J, 2009. 182(7): 4005-16.

43. Mishra B.B, et al. MyD88-deficient mice exhibit decreased parasite-induced immune responses but reduced disease severity in a murine model of neurocysticercosis. Infect Immun, 2009. 77(12): 5369-79.

44. Jorge I Alvarez; Bibhuti B Mishra; Uma Mahesh Gundra; Pramod K Mishra; Judy M Teale. Mesocestoides corti intracra- nial infection as a murine model for neurocysticercosis. Parasitology 2010;137(3):359-72.

45. Verma A, et al. Toll-like receptor 4 polymorphism and its association with symptomatic neurocysticercosis. J Infect Dis, 2010. 202(8): 1219-25.

46. Rodriguez-Sosa M, et al. A STAT4-dependent Th1 response is required for resistance to the helminth parasite Taenia crassiceps. Infect Immun, 2004. 72(8): 4552-60.

47. Zhang M, et al. Toll-like receptor (TLR) 2 and TLR4 deficiencies exert differential in vivo effects against Schistosoma japonicum. Parasite Immunol, 2011. 33(4): 199-209.

48. Dissanayake $S$ and Shahin A. Induction of interferon-gamma by Taenia crassiceps glycans and Lewis sugars in naive BALB/c spleen and peritoneal exudate cells. Molecular immunology, 2007. 44(7): 1623-30.

49. Uddin J, et al. Mechanisms regulating monocyte CXCL8 secretion in neurocysticercosis and the effect of antiparasitic therapy. Journal of immunology, 2010. 185(7): 4478-84.

50. Mills G.L, Taylor D.C, and Williams J.F. Lipid composition of metacestodes of Taenia taeniaeformis and lipid changes during growth. Mol Biochem Parasitol, 1981. 3(5): 301-18. 